\title{
CONSTRUÇÃO E CARACTERIZAÇÃO DE UM FOTÔMETRO DESTINADO AO USO DE AULAS EXPERIMENTAIS DE QUÍMICA SOBRE A LEI DE BEER-LAMBERT
}

\author{
A. F. MOREIRA ${ }^{1 *}$, S. R. B. SANTOS ${ }^{2}$ e A. G. COSTA JUNIOR ${ }^{1}$ \\ ${ }^{1}$ Laboratório de Instrumentação, Sistemas de Controle e Automação (LINSCA) \\ ${ }^{2}$ Licenciatura em Química \\ Instituto Federal de Educação, Ciência e Tecnologia da Paraíba - IFPB, campus João Pessoa \\ alexfmgt@hotmail.com*
}

Artigo submetido em fevereiro/2016 e aceito em março/2016

DOI: $10.15628 /$ holos. 2016.4016

\section{RESUMO}

Este artigo apresenta o desenvolvimento de um fotômetro de baixo custo para aulas experimentais de química, através da medição dos valores de absorbância de determinadas concentrações de substâncias, para a comprovação da lei de Beer-Lambert. A aquisição de sinais do protótipo do fotômetro foi realizada através do uso de um microcontrolador Arduino Uno e o tratamento dos dados é realizado no MS-Excel, através do programa
PLX-DAQ que recebe os dados do microcontrolador de forma automática. Os resultados experimentais apresentados demonstram o bom funcionamento do fotômetro construído, em que se utilizam materiais de fácil aquisição no mercado para que possam ser construídos para aulas experimentais, sem a necessidade de utilizar grandes recursos financeiros para a aquisição de equipamentos comerciais.

PALAVRAS-CHAVE: Absorbância, Arduino, Experimentos de química, Fotômetro, Lei de Beer-Lambert.

\section{CONSTRUCTION AND CHARACTERIZATION OF A PHOTOMETER FOR THE LESSONS OF EXPERIMENTAL CHEMICAL ABOUT BEER-LAMBERT LAW}

\begin{abstract}
This paper presents the development of a low cost photometer for experimental lessons of chemistry, by measuring the absorbance values of certain concentrations of substances, for attesting the BeerLambert law. The signal acquisition of prototype of photometer was performed by using an Arduino Uno microcontroller and the processing is performed in MS-
\end{abstract}

Excel through PLX-DAQ program that receives data from the microcontroller automatically. Experimental results demonstrate the proper functioning of the photometer built, in which use easily obtainable materials in the market so they can be built for experimental classes without the need to use large financial resources for the purchase of commercial equipment.

KEYWORDS: Absorbance, Arduino, Beer-Lambert law, Chemistry experiments, photometer. 


\section{INTRODUÇÃO}

Uma das grandes dificuldades no ensino é fazer a ponte entre o conhecimento teórico e suas aplicações práticas, gerando, muitas vezes, a apatia do aluno com o conhecimento abordado. Os conceitos quando vistos somente textualmente, não são plenamente compreendidos pelos discentes, surgindo lacunas de conhecimento na formação educacional dos mesmos. Desse modo, a realização de atividades experimentais é extremamente importante no processo de ensinoaprendizagem, contribuindo, significativamente, para um melhor aproveitamento dos conhecimentos socializados em sala de aula e, consequentemente, uma compreensão mais abrangente da ciência.

Ensinar não é simplesmente apresentar conhecimento são aluno e aguardar que este, em um piscar de olhos, tenha domínio sobre o conteúdo informado. Freire (2008) ressalta que ensinar não é transferir o conhecimento, mas criar as possibilidades para a sua própria produção, ou a sua construção.

Giani (2010) afirma que o professor deve estar bem preparado e motivado para o desenvolvimento de atividades experimentais, para que possa explorar as suas potencialidades, e desenvolver aulas experimentais com mais frequência e com melhores resultados.

Estas aulas experimentais nem sempre são simples e a falta de estrutura é um problema crônico que gera grandes dificuldades para o desenvolvimento destas práticas experimentais em algumas escolas. A falta de equipamentos, por exemplo, inviabiliza muito destas práticas de ensino em laboratórios ou mesmo em salas de aula. Uma saída alternativa para este problema é a utilização de equipamentos construídos com materiais de baixo custo e/ou de sucatas, para a realização dos experimentos que envolvam os conteúdos apresentados em teoria, em que diversos trabalhos foram propostos para a realização de atividades experimentais de química (SWEET, 2004; VRTACNIK \& GROS, 2005; YEH \& TSENG, 2006; GONG et al, 2009; MOTA, 2010; ALBERT et al, 2012).

O objetivo desse artigo é apresentar a construção de um protótipo de baixo custo, que permita a apresentação de conceitos de espectroscopia por meio da confirmação da Lei de BeerLambert utilizando-se de medidas de absorbâncias de um conjunto de soluções padrão, (SWINEHART, 1962; KOCSIS et al, 2006), através do uso do microcontrolador Arduino UNO e do PLX-DAQ, que realiza a interface entre o Arduino e o software Excel ${ }^{\circledR}$, tornando o protótipo automatizado.

Este artigo está dividido desse modo. Na Seção 2 é realizada uma breve introdução à espectrofotometria. Na Seção 3 é apresentado o protótipo desenvolvido e na Seção 4, os resultados obtidos com sua utilização. Por fim, na Seção 5, as considerações finais deste trabalho.

\section{UMA BREVE INTRODUÇÃO À ESPECTROFOTOMETRIA}

A espectrofotometria e os métodos espectrofotométricos amplamente empregados em química analítica referem-se às medidas das interações entre a radiação eletromagnética e a matéria, com o objetivo de identificar um componente (analito) presente em uma amostra, em um procedimento qualitativo, ou determinar a quantidade deste analito, em um procedimento 
quantitativo. Ao conjunto de radiações eletromagnéticas se denomina espectro eletromagnético. O espectro eletromagnético pode ser dividido em várias regiões de acordo com características da radiação, abrangendo um intervalo muito amplo de comprimentos de ondas e de frequências, conforme ilustrado na Figura 1. Esse intervalo é empregado para estabelecer as divisões dos métodos espectroscópicos.

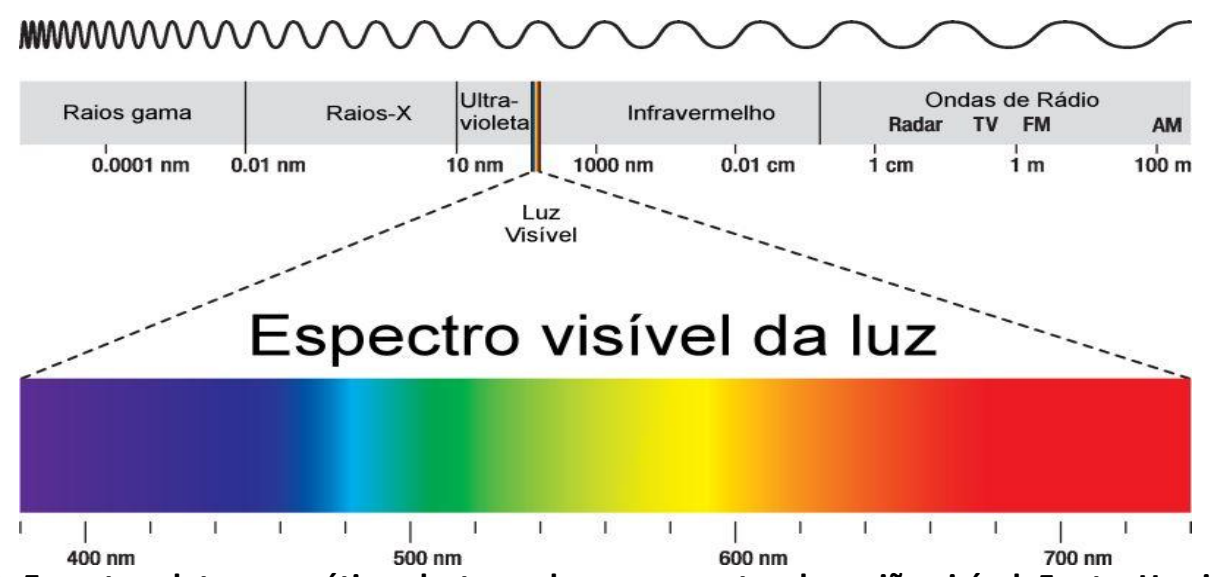

Figura 1: Espectro eletromagnético, destacando componentes da região visível. Fonte: Harris, 2008.

As determinações espectrofotométricas da quantidade de matéria normalmente se baseiam na absorção da radiação ultravioleta, do visível ou do infravermelho por uma solução (SKOOG et al, 2009).

Quando a radiação eletromagnética atinge uma amostra, parte de sua energia pode ser absorvida, promovendo a excitação de espécies químicas (OHWEILER, 1976; VOGEL et al, 2002; HARRIS, 2008). A absorção da radiação depende da estrutura das espécies atômicas, ou moleculares, envolvidas. Quando um feixe de radiação eletromagnética, $P_{0}$, atravessa uma amostra contendo o analito, algumas frequências da radiação podem ser seletivamente retidas pelo material, de modo que apenas um feixe de radiação, $P$, de menor intensidade que o feixe incidente é transmitido pela amostra, como ilustrado na Figura 2 (VOGEL et al, 2002; SKOOG et al, 2009).

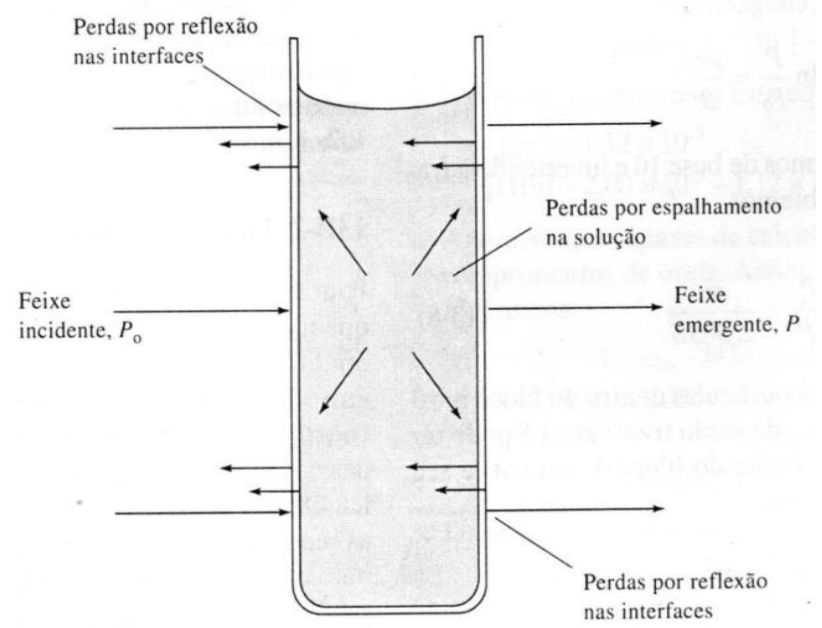

Figura 2: Processo de absorção de radiação por um analito. Fonte: Skoog et al, 2009.

Quando as absorções da radiação ocorrem na região visível do espectro eletromagnético, as amostras apresentam cor. A cor que um material apresenta quando a luz branca (conjunto das radiações eletromagnéticas da região visível) os atinge, corresponde à radiação transmitida e que, 
portanto, não foi absorvida pelo material, em que pode ser dito que a cor absorvida é a cor complementar da cor transmitida. A união da cor transmitida com a cor absorvida recompõe a cor branca. A Tabela 1 ilustra os intervalos de comprimento de onda correspondente a cada cor (OHWEILER, 1976; HARRIS, 2008).

Tabela 1: Comprimentos de onda correspondente à absorção máxima, e as relações entre a cor visível e a cor da radiação absorvida. Fonte: Ohweiler, 1976.

\begin{tabular}{c|c|c}
\hline $\begin{array}{c}\text { Intervalo de comprimento } \\
\text { de onda }(\mathrm{nm})\end{array}$ & Cor absorvida & $\begin{array}{c}\text { Cor complementar } \\
\text { (observada) }\end{array}$ \\
\hline $380-435$ & Violeta & Verde-Amarelo \\
$435-480$ & Azul & Amarelo \\
$480-490$ & Azul-verde & Laranja \\
$490-500$ & Verde-azul & Vermelho \\
$500-560$ & Verde & Púrpura \\
$560-580$ & Verde-amarelo & Violeta \\
$580-595$ & Amarelo & Azul \\
$595-650$ & Laranja & Azul-verde \\
$650-780$ & Vermelho & Verde-azul \\
\hline
\end{tabular}

\subsection{Lei de Beer-Lambert}

A espectrometria de absorção molecular está baseada na medida da transmitância, $T$, ou da absorbância, $A$, de soluções contidas em células transparentes, tendo um caminho óptico, $b$, dado em $\mathrm{cm}$. Além disso, a concentração $c$ de um analito absorvente está relacionada linearmente à absorbância, conforme indicada pela Equação (1), que é a representação matemática da lei de Beer-Lambert (HARRIS, 2008; SKOOG et al, 2009; SKOOG et al, 2015):

$$
A=-\log T
$$

A transmitância é definida como a fração da luz original que passa pela amostra, sendo calculada como (HARRIS, 2008):

$$
T=\frac{P_{0}}{P}
$$

em que, $P_{0}$ é a energia radiante que atinge a amostra, que está dentro de uma cubeta, com espessura $b$, e $P$, é a energia radiante do feixe que sai do outro lado da amostra.

Quanto maior for o caminho através do qual a luz atravessa, haverá mais centros absorventes, implicando em uma atenuação maior do feixe incidente (HARRIS, 2008). De acordo com a Lei de Beer-Lambert, a absorbância é diretamente proporcional à concentração da espécie absorvente, $c$ e ao caminho óptico, $b$, do meio absorvente, de acordo com (HARRIS, 2008; SKOOG et al, 2015):

$$
A=e b c
$$

Na equação de Beer-Lambert, $e$ é a constante de absortividade molar da substância absorvente, dada em mol. $L^{-1} \cdot \mathrm{cm}^{-1}$. A absorbância, a transmitância e a potência da radiação incidente, apresentadas pelas Equações (1)-(3), se relacionam como apresentado na Equação (4): 


$$
A=-\log T=\log \frac{P_{0}}{P}=e b c
$$

\subsection{Fotômetros}

Os fotômetros são equipamentos simples, práticos e baratos quando comparados com espectrofotômetros. As medidas realizadas nestes equipamentos exploram faixas estreitas da região espectral visível. Em geral, os fotômetros são constituídos de uma fonte de radiação eletromagnética visível que pode emitir luz policromática (branca) e que exige um seletor de comprimento de onda (filtro ou sistema monocromador) ou monocromática, no caso de uso de LEDs; um compartimento para adaptação da amostra; um fotodetector e uma etapa de condicionamento do sinal. Estes dispositivos podem ser arranjados de diversas formas, muito embora existam apenas duas classificações: os de feixe único, e os de feixe duplo, sendo o primeiro ilustrado na Figura 3 (SKOOG et al, 2009).

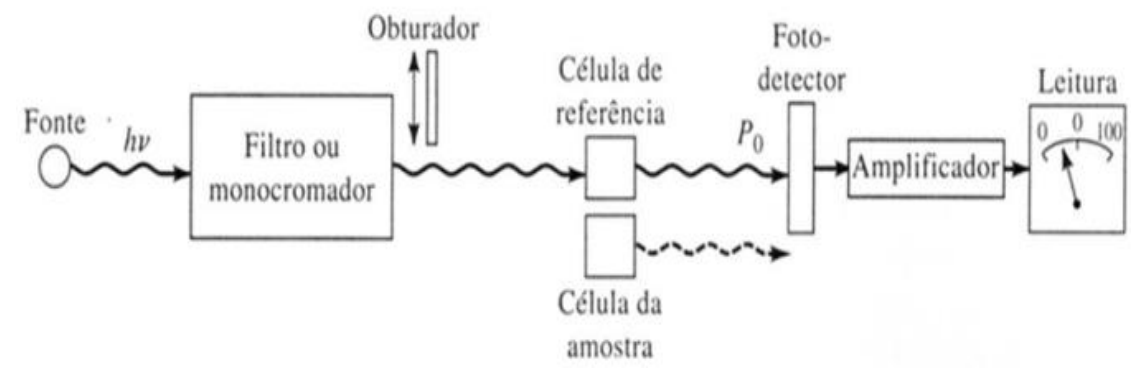

Figura 3. Diagrama básico de um fotômetro de feixe único. Fonte: SKOOG et al, 2007.

A potência radiante nos fotômetros é determinada por um detector de radiação que converte a energia radiante em sinal elétrico $S$. Em geral, $S$ é uma tensão ou corrente elétrica que é idealmente proporcional à potência radiante, ou seja:

$$
S=k P
$$

em que $k$ é uma constante, e $P$, a energia radiante (SKOOG et al, 2007).

\section{MATERIAIS E MÉTODOS}

A plataforma de desenvolvimento Arduino é uma plataforma de prototipagem eletrônica com sistemas digitais que quando conectados a sensores e a atuadores, são capazes de medir variáveis no ambiente físico, realizar cálculos numéricos, e tomar decisões lógicas no ambiente computacional gerando novas variáveis no ambiente físico (ARAUJO et al, 2012).

O Arduino faz parte do conceito de hardware e de software livre, e está aberto para uso e contribuição por toda a sociedade. Pelo fato do Arduino ser open source, e ser de fácil aquisição, existem diversos fóruns na Internet que oferecem suporte aos usuários e aos projetistas, assim como, o compartilhamento de ideias e de projetos com outros desenvolvedores. Para o desenvolvimento do projeto, foi utilizada a plataforma Arduino Uno, baseada no microcontrolador ATmega328, possuindo 14 terminais de entrada/saída (06 podem ser usados como saída PWM), 
06 entradas analógicas, um oscilador a cristal de frequência de $16 \mathrm{MHz}$, uma conexão USB, entre outros detalhes (ARDUINO, 2015).

Para a montagem do fotômetro utilizou-se a configuração de feixe único, ilustrado na Figura 3. No protótipo desenvolvido, a fonte de radiação eletromagnética é o LED RGB que emite nos comprimentos de onda de $470 \mathrm{~nm}$ (azul), $525 \mathrm{~nm}$ (verde) e $625 \mathrm{~nm}$ (vermelho), com larguras de banda à metade da intensidade máxima, menores que $35 \mathrm{~nm}$, dispensando assim o uso de um filtro monocromador (KOUHI, 2015).

Além do LED RGB, foram utilizados uma bateria de $9 \mathrm{~V}$, um fototransistor, resistores, um amplificador operacional 741, um diodo zener, chaves lida-desliga, uma cubeta de acrílico quadrada de $10 \mathrm{~mm}$ de caminho óptico, um microcontrolador Arduino UNO para aquisição de sinais e o programa PLX-DAQ, que realiza a interface entre o microcontrolador e o software MSExcel $^{\circledR}$.

O protótipo consiste em dois circuitos, divididos em um circuito da alimentação da fonte de radiação e um circuito para a aquisição de sinais. O circuito elétrico da fonte de radiação do fotômetro proposto está ilustrado na Figura 4 em que o LED RGB está representado por D4, D6 e D7. O circuito de aquisição de sinais do fotômetro é ilustrado na Figura 5.

Na Tabela 2 estão listados os materiais utilizados, de acordo com os componentes ilustrados nos circuitos elétricos das Figuras 4 e 5.

Tabela 2. Componentes utilizados na montagem do protótipo.

\begin{tabular}{c|c|c}
\hline Elemento do circuito & Descrição & Valor \\
\hline B1 & Bateria & $9,0 \mathrm{~V}$ \\
SW1 & Chave comutadora & -- \\
R4 & Resistor & $470 \Omega$ \\
R7 & Resistor & $100 \Omega$ \\
R6 & Resistor & $1 \mathrm{k} \Omega$ \\
R1, R2, R5, R8, R9 & Resistores & $10 \mathrm{k} \Omega$ \\
D1 & Diodo Zener & $3,3 \mathrm{~V}(0,5 \mathrm{~W})$ \\
D5 & Diodo Zener & $6,2 \mathrm{~V}(0,5 \mathrm{~W})$ \\
D2 & LED Verde & --- \\
D4, D6, D7 & LED RGB \\
Q1 & Fototransistor & -- \\
Arduino & Fonte do Arduino & -- \\
U1 & Amplificador Operacional 741 & $5,0 \mathrm{~V}$ \\
\hline
\end{tabular}

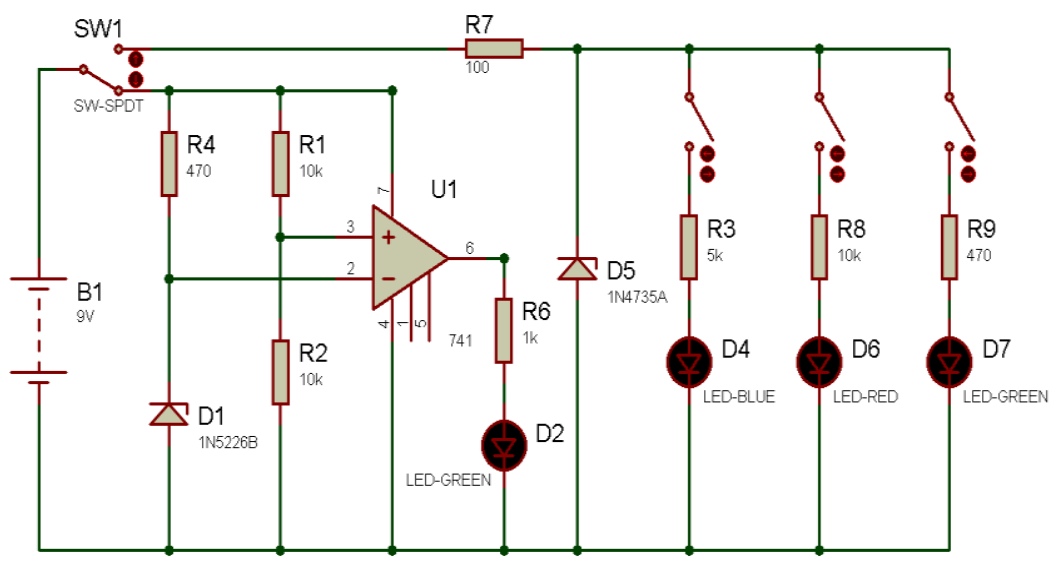

Figura 4. Circuito elétrico da fonte de radiação do fotômetro. 


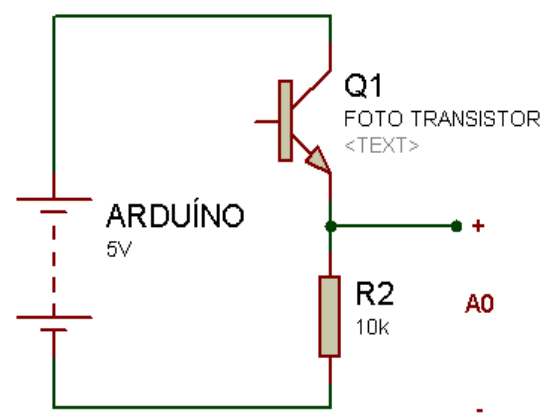

Figura 5. Circuito elétrico aquisição de sinais do fotômetro.

O protótipo do fotômetro de feixe único montado é ilustrado na Figura 6, em que os circuitos de alimentação da fonte de radiação e de aquisição de sinais estão compactados em um circuito impresso, representado pelo número 1; o compartimento da amostra está representado pelo número 2; as chaves de comutação que selecionam o comprimento de onda da fonte de emissão de luz estão representadas pelo número 3; o circuito de aquisição de sinais é alimentado pelos bornes fêmeas, representado pelo número 4.

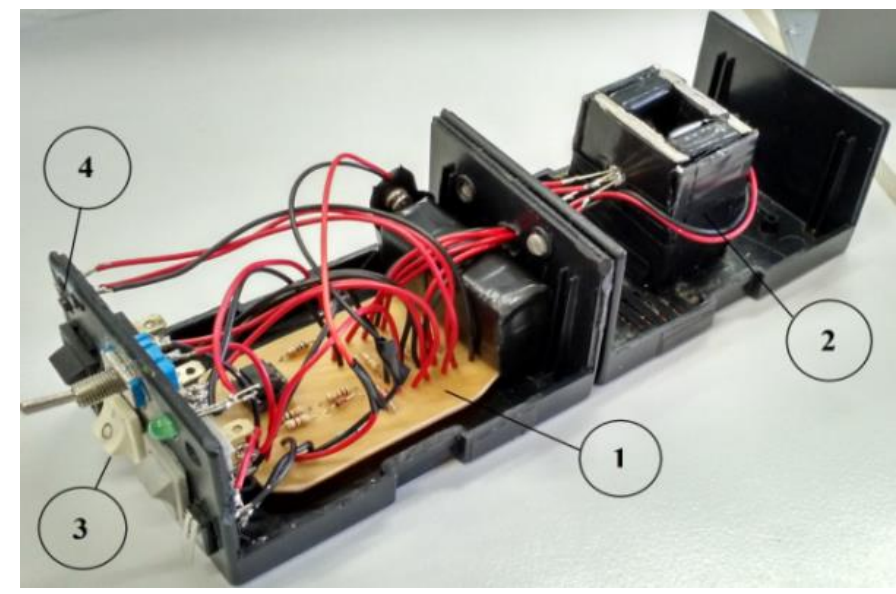

Figura 6. Protótipo do fotômetro de feixe único montado.

Para a avaliação do desempenho do fotômetro desenvolvido foram realizadas medidas de absorbância de soluções e sulfato de níquel $\left(\mathrm{NiSO}_{4}\right)$, na cor verde, com concentrações, em mol/L, de $0.4 \times 10^{-4}, 0.6 \times 10^{-4}, 0.8 \times 10^{-4}$ e $0.1 \times 10^{-4}$. Para tanto foi registrado o sinal do branco (água destilada) de modo a se obter o valor de $P_{0}$, relacionado à potência radiante máxima que atinge $o$ detector sem a influência da absorbância do analito. Logo após foram registrados os sinais das soluções padrão de sulfato de níquel relacionados à potência radiante transmitida e atenuada após atravessar a solução absorvente. A partir destes sinais é possível determinar a absorbância das soluções por aplicação da Equação (4).

De acordo com a Tabela 1, se a cor observada é o verde-azul, a radiação da região espectral vermelha está sendo absorvido. Desse modo, o LED vermelho foi selecionado como fonte de radiação visto que a solução usada possui cor esverdeada. Para cada concentração da solução, foram realizadas dez medições de tensões elétricas na saída do protótipo, através do Arduino Uno. Com a utilização do software PLX-DAQ, os valores obtidos foram repassados ao MS-Excel, em tempo real, aplicando-se a Equação 2 para o cálculo da transmitância, e a Equação (1) para o 
cálculo da absorbância, em que $P_{0}$ é a tensão elétrica da amostra com água destilada, e $P$ é a tensão elétrica de cada amostra de concentração do $\mathrm{NiSO}_{4}$.

\section{RESULTADOS E DISCUSSÕES}

Os resultados das aquisições de sinais obtidas através do uso com Arduino são ilustrados na Tabela 3. Para cada concentração, foram calculadas as médias e os desvios padrões das tensões elétricas, além da absorbância das amostras.

Tabela 3. Valores das tensões elétricas e das absorbâncias no fotômetro em função da concentração das soluções.

\begin{tabular}{c|c|c|c|c|c}
$\begin{array}{c}\text { Concentração } \\
(\text { mol } / \text { L) }) \text { 10 } \mathbf{0}^{-3}\end{array}$ & Amostra $\boldsymbol{P}_{\mathbf{0}}$ & $\begin{array}{c}\mathbf{0 , 0 4}(\text { Amostra } \\
\boldsymbol{P}_{\mathbf{1}} \text { ) }\end{array}$ & $\begin{array}{c}\mathbf{0 , 0 6} \\
\left(\text { Amostra } \boldsymbol{P}_{\mathbf{2}} \text { ) }\right.\end{array}$ & $\begin{array}{c}\mathbf{0 , 0 8} \text { (Amostra } \\
\boldsymbol{P}_{\mathbf{3}} \text { ) }\end{array}$ & $\begin{array}{c}\mathbf{0 , 1} \\
\left(\text { Amostra } \boldsymbol{P}_{\mathbf{4}} \text { ) }\right.\end{array}$ \\
\hline Tensão elétrica (V) & $3,38 \pm 0,010$ & $3,02 \pm 0,013$ & $2,87 \pm 0,012$ & $2,73 \pm 0,014$ & $2,58 \pm 0,014$ \\
\hline Absorbância & - & 0,048 & 0,071 & 0,093 & 0,117
\end{tabular}

A partir dos valores de tensão elétrica registrados para as soluções padrão foram calculadas as absorbâncias das soluções. A curva analítica apresentando a absorbância em função da concentração das soluções, bem como a linha de tendência por regressão linear estimada são ilustradas na Figura 7.

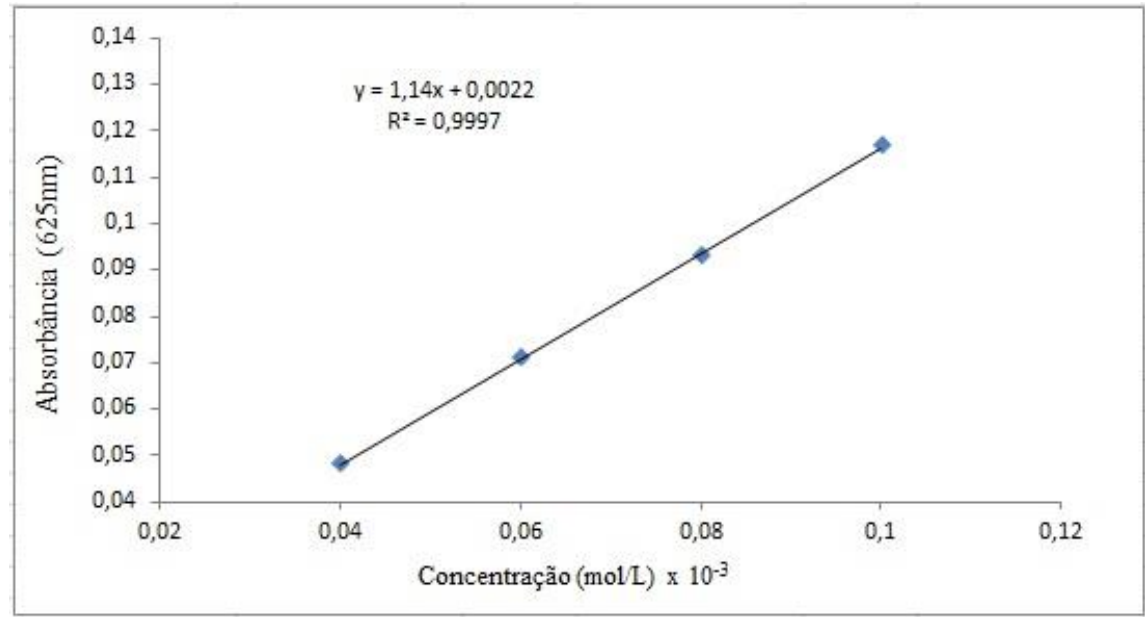

Figura 7. Curva da absorbância em função da concentração das soluções.

Como pode ser observado da Figura 7, o aumento das absorbâncias é linear com o aumento da concentração da solução, o que comprova a Lei de Beer-Lambert. Também se observa que a equação de reta possui um valor do coeficiente de determinação $R^{2}$ próximo a unidade, ou seja, a equação encontrada consegue explicar os valores observados. A equação de reta encontrada é $y=1,14 x+0,0022$ em que $y$ é a absorbância, e $x$ é a concentração das amostras.

\section{CONSIDERAÇÕES FINAIS}

O protótipo de fotômetro de feixe único pode ser construído com materiais de baixo custo e de fácil aquisição no mercado, sendo uma alternativa para demonstrações, em forma experimental, de alguns dos conceitos apresentados em sala de aula referentes à 
espectrofotometria e à Lei de Beer-Lambert. O custo aproximado do projeto do fotômetro é de R\$ 60 , que possui um custo bem menor quando comparado com um comercial que custa em torno de $R \$ 500$.

A aquisição de sinais de forma automática também pode proporcionar uma melhor leitura, onde não há intervenção humana, diminuindo os erros de medições e tornando o aparelho mais confiável. Entretanto, o registro dos sinais analíticos $P_{0}$ e $P$ pode ser realizado com um simples voltímetro de modo que o processamento digital de sinais não é fator limitante da aplicação do fotômetro.

O fotômetro desenvolvido demonstrou ser um instrumento confiável no registro de sinais analíticos com o propósito de determinar as concentrações de amostras de sulfato de níquel e, portanto, útil no ensino de espectrofotometria para alunos de Química. Os próximos passos serão estender as aplicações do fotômetro para aulas práticas de Química e realizar a avaliação analítica de amostras reais.

\section{REFERÊNCIAS}

1. ALBERT D. R. et al. A low-cost quantitative absorption spectrophotometer. Journal of Chemical Education, v.89, n.11, p. 1432-1435, 2012.

2. ARAUJO, I. B. Q. et al. Desenvolvimento de um protótipo de automação predial/residencial utilizando a plataforma de prototipagem eletrônica Arduino. Anais: XL Congresso Brasileiro de Educação em Engenharia (Cobenge), Belém, UFPA, 2012.

3. ARDUINO. Disponível em: <http://arduino.cc/>. Acesso em dezembro de 2015.

4. FREIRE, P. Pedagogia de Oprimido. São Paulo: Paz e Terra, 1994.

5. GIANI, K. Experimentação no ensino de ciências: possibilidades e limites na busca de uma aprendizagem significativa, 2010. Dissertação (Mestrado em Ensino de Ciências) - PósGraduação em Ensino de Ciências da Universidade de Brasília, Brasília, 2010.

6. GONG, W. et al. A simple, low-cost double beam spectrophotometer for colorimetric detection of nitrite in seawater. IEEE Sensor Journal, v.09, n.07, p. 862-869, 2009.

7. HARRIS, D. A. Química Analítica Quantitativa. Rio de Janeiro: LTC, 2008.

8. KOCSIS, L. et al. The modified Beer-Lambert law revisited. Physics in Medicine and Biology, v.51, n. 05, 2006.

9. KOUHI. LED Lamps Specification Datasheet. Disponível em: <http http://www.leds.com.hk/pdf/KTL050RGBCI\%20CC.pdf>. Acesso em dezembro de 2015.

10. MOTA, F. Desenvolvimento de um fotômetro com fins didáticos, 2010. Dissertação (Mestrado em Química) - Instituto de Ciências Exatas da Universidade Federal do Amazonas, Manaus, 2010.

11. OHWEILER, O. A. Química Analítica Quantitativa. Rio de Janeiro: LTC, 1976.

12. SKOOG, D. A. et al. Princípios de Análise Instrumental. Porto Alegre: Bookman / Grupo A, 2009.

13. SKOOG, D. A. et al. Fundamentos da Química Analítica. São Paulo: Cengage, 2015.

14. SWEET, F. Low-cost vacuum desiccator. Journal of Chemical Education, v.81, n.10, p. 1500, 2004. 
15. SWINEHART, D. F. The Beer-Lambert law. Journal of Chemical Education, v.39, n.7, p.333, 1962.

16. VOGEL, A. L. et al. Análise Química Quantitativa. Rio de Janeiro: LTC, 2002.

17. VRTACNIK, M.; GROS, N. A small-scale low-cost gas chromatograph. Journal of Chemical Education, v.82, n.02, p. 291, 2005.

18. YEH, T. S.; TSENG, S. S. A low cost LED based spectrometer. Journal of the Chinese Chemical Society, v.53, n.05, p. 1067-1072, 2006. 